\title{
Quantum graphs and dimensional crossover: the honeycomb
}

\author{
Riccardo Adami ${ }^{{ }^{*} \dagger}$, Simone Dovetta ${ }^{1}$, Alice Ruighi ${ }^{1}$ \\ ${ }^{1}$ Dipartimento di Scienze Matematiche "G.L. Lagrange", Politecnico di Torino, Corso Duca degli Abruzzi, 24, \\ 10129 Torino, Italy \\ *Email address for correspondence: riccardo.adami@polito.it \\ Communicated by Luca Formaggia \\ Received on 01 30, 2019. Accepted on 05 17, 2019.
}

\begin{abstract}
We summarize features and results on the problem of the existence of Ground States for the Nonlinear Schrödinger Equation on doubly-periodic metric graphs. We extend the results known for the two-dimensional square grid graph to the honeycomb, made of infinitely-many identical hexagons. Specifically, we show how the coexistence between one-dimensional and two-dimensional scales in the graph structure leads to the emergence of threshold phenomena known as dimensional crossover.
\end{abstract}

Keywords: periodic graphs, nonlinear Schrödinger, threshold phenomena, Sobolev inequality

AMS subject classification: 35Q55, 35R02

\section{Introduction}

In the last decade there has been a dramatic increase in the study of the dynamics of systems on metric graphs, or networks. This is mainly due to two different issues: first, the extensive use of mathematics in topics traditionally confined to a more qualitative approach (e.g. biology, social sciences, economics); second, the flexibility and the simplicity of networks as a mathematical environment to model phenomena occurring in the actual world.

Networks enter in the description of evolutionary phenomena on branched structures, namely, onedimensional complexes made of edges, either finite or infinite, meeting at special points called vertices. Edges and vertices define the topology of the graph. The metric structure is defined by associating to every edge a length and then an arclength. This is easily accomplished by associating to every edge $e$ a coordinate $x \in\left[0, \ell_{e}\right]$, where $\ell_{e}$ is the length of the edge.

Such a scheme applies to signals propagating in networks, circuits, and to more recent scientific and technological challenges of the new emerging field of research called Atomtronics.

The first appearance of metric graphs in the mathematical modeling of natural systems dates back to 1953 and is due to Ruedenberg and Scherr [1], who modeled a naphtalene array as a network of edges and vertices arranged in a hexagonal lattice, like a honeycomb. Then, a Hamiltonian operator representing the quantum energy of the system was defined on such a structure, and its spectrum was computed in order to deduce the possible values of the energy of the valence electrons. The paper is not only a milestone in physical chemistry, but it also introduces some important mathematical tools like the so-called Kirchhoff's conditions at the vertices of the graph, and it opens the research field of quantum graphs. Dealing with a standard quanto-mechanical system, the model is governed by a linear equation, i.e. the Schrödinger equation of the system.

Since then, the use of metric graphs has become widespread in the literature, exiting the realm of quantum mechanics and extending to electromagnetism, acoustics, and many others physically relevant contexts. However, most of the models were linear. The first systematic introduction to nonlinear dynamics on graphs was given by Ali Mehmeti [2] in a nowadays classical treatise published in 1994, but

\footnotetext{
${ }^{\dagger}$ We acknowledge that the present research has been partially supported by MIUR grant Dipartimenti di Eccellenza 2018-2022 (E11G18000350001)
} 
one had to wait about three decades to see the analysis of the dynamics of a specific nonlinear model, first given in [3] and concerning the effect of the impact of a fast soliton of the Nonlinear Schrödinger Equation (NLSE) on the vertex of an infinite star-graph. After this result, the research on the NLSE on graphs underwent an important development, especially because of great technical advances on the study of the mathematical aspects of the nonlinear Schrödinger Equation (especially following the seminal papers by Keel and Tao [4] and by Kenig and Merle [5]) from one side, and because of the rapid evolution of the technology of Bose-Eintein condensates (BEC) from the other, and in particular of the new accomplishments in the construction of traps of various shapes, to be used in BEC experiments.

In order to motivate the mathematical problem we are dealing with, let us be more specific on this point. A Bose-Einstein condensate is a system of a large (from thousands to millions) number of identical bosons, usually magnetically and/or optically confined in a spatial region, called trap. As predicted by Bose [6] and Einstein [7], under a prescribed value of the temperature, called "critical value", the system collapses into a very peculiar and non-classical state, in which:

- Every particle acquires an individual wave function (which is in general not the case for manybody systems, that are given a collective wave function only).

- The wave function is the same for all particles, and is called wave function of the condensate.

- The wave function of the condensate solves the following variational problem:

$$
\min _{u \in H^{1}(\Omega), \int|u|^{2}=N} E_{G P}(u)
$$

where

- $E_{G P}$ is the Gross-Pitaevskii energy (GP) functional, namely

$$
E_{G P}(u)=\|\nabla u\|_{L^{2}(\Omega)}^{2}+8 \pi \alpha\|u\|_{L^{4}(\Omega)}^{4}
$$

( $\alpha$ is the scattering length of the two-body interaction between the particles in the condensate);

$-\Omega$ is the trap where the condensate is confined;

$-N$ is the number of particles in the condensate;

- provided it exists, the minimum corresponds to a standing wave for the Gross-Pitaevskii Nonlinear Schrödinger Equation

$$
i \partial_{t} \psi(t, x)=-\Delta \psi(t, x)+32 \pi \alpha|\psi(t, x)|^{2} \psi(t, x) .
$$

Then it becomes an important issue to solve the problem of minimizing the functional (2) under the constraint $\int_{\mathcal{G}}|u|^{2} d x=\mu$ given in (1). As one might expect, the result heavily depends on $\Omega$, not only for what concerns the actual shape of the minimizer, but also for the sake of its mere existence. It is indeed this last issue that has been mostly studied during the last years, and will be the subject of the present note.

\subsection{Existence of ground states: results}

From now on, we consider a metric graph $\mathcal{G}$ and the NLS energy functional defined as

$$
E(u, \mathcal{G})=\frac{1}{2} \int_{\mathcal{G}}\left|u^{\prime}\right|^{2} d x-\frac{1}{p} \int_{\mathcal{G}}|u|^{p} d x
$$

The first term is called kinetic term, as it represents the kinetic energy associated to the system, while the second is the nonlinear term.

The main difference of (3) with respect to the GP energy (2) is that in (3) a more general nonlinearity power is considered instead of the only case $p=4$, but we restrict to the so-called focusing case, where the nonlinear term has a negative sign, and encodes the fact that the two-body interaction between the particles is attractive. 
Owing to the choice of the sign, it is clear that there is a competition between the two terms: the kinetic term favours widespread signals, while the nonlinear term prevents the minimizers from dispersing too much. When a minimizer exists, it always realizes a compromise between the two terms and the two corresponding tendencies: spreading or squeezing.

We study the problem of minimizing the energy (3) with the constraint of constant mass, namely

$$
\|u\|_{L^{2}(\mathcal{G})}^{2}=\int_{\mathcal{G}}|u|^{2} d x=\mu>0
$$

We shall use the notation

$$
\mathcal{E}(\mu):=\inf _{u \in H_{\mu}^{1}(\mathcal{G})} E(u, \mathcal{G})
$$

and introduce the ambient space

$$
H_{\mu}^{1}(\mathcal{G}):=\left\{u \in H^{1}(\mathcal{G}):\|u\|_{L^{2}(\mathcal{G})}^{2}=\mu\right\}
$$

We call ground state at mass $\mu$ or, for short, ground state, every minimizer of (3) among all functions sharing the same mass $\mu$.

First of all, it is well-known [8-10], that in the case of the real line, and provided that $2<p<6$, the compromise between kinetic and nonlinear term that gives rise to a ground state is realized for every $\mu$ by the soliton

$$
\phi_{\mu}(x)=\mu^{\alpha} \phi_{1}\left(\mu^{\beta} x\right), \quad \alpha:=\frac{2}{p-2}, \beta:=\frac{p-2}{6-p},
$$

where the prototype soliton is denoted by $\phi_{1}$ and equals

$$
\phi_{1}(x):=C \operatorname{sech}(c x) .
$$

In the case of a real half-line $\mathbb{R}^{+}$, by elementary symmetry arguments one can immediately realize that a solution exists for every value of the mass $\mu$ and it coincides with a half-soliton with the maximum at the origin, possibly multiplied by a phase factor.

Despite the result for the half-line and for the line (i.e. a pair of half-lines), for the graph made of three half-lines meeting one another at a single vertex (i.e. a star graph) it has been proven that there is no ground state, irrespectively of the choice of $\mu$ ( [11]). Starting from this negative result, the problem of ensuring (or excluding) the existence of ground states for the NLS on graphs gained some popularity in the community, and some general results were found, isolating a key topological condition ( [12]), studying in detail particular cases ( [13-15]), dealing with compact graphs ( $[16,17])$, introducing concentrated nonlinearities ( [18-21]), focusing on the more challenging $L^{2}$-critical case (i.e. $p=6$ [22]). More recently, also some pioneering investigations of nonlinear Dirac equations has been initiated ( $[23,24])$.

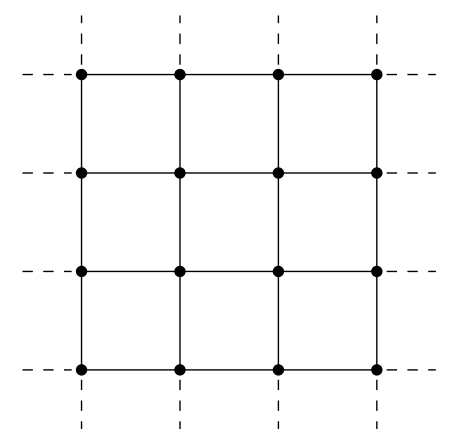

Figure 1. the two-dimensional square grid. 
The analysis of NLS equations on periodic graphs has been developed for instance in ( [25-27]), and a systematic discussion of the problem of ground states for periodic graphs has been carried out in [28], however here we shall focus on a particular phenomenon highlited in [29] and called dimensional crossover. Investigating the problem of proving the existence or the nonexistence of ground states for the NLS on the regular two-dimensional square grid (see Figure 1), it was found that three different regimes come into play:

1. if $2<p<4$, then a ground state exists for every value $\mu$ of the mass;

2. if $p>6$, then there is no ground state irrespectively of the value chosen for the mass;

3. if $p=6$, then there is a particular value of the mass, called critical mass and denoted by $\mu^{*}$, such that the infimum of the energy passes from 0 to $-\infty$ as the mass exceeds $\mu^{*}$, and ground states never exist for any value of the mass;

4. if $4 \leq p<6$, then there is a particular value of the mass, $\mu_{p}$, such that ground states exist only beyond $\mu_{p}$.

Now, points 1 and 2 are common to what one finds in the problem of the ground states in $\mathbb{R}$ and $\mathbb{R}^{2}$. The transition of the actual value of the infimum of the energy as in point 3 is characteristic of one-dimensional domains, in particular of quantum graphs made of a compact core and a certain number of half-lines.

What really distinguishes the case of the grid graph from the previously studied cases of quantum graphs is point 4, where an unprecedented behaviour is detected for nonlinearity powers ranging from 4 to 6 . Here power 4 is meaningful since it is the critical power for two-dimensional problems. Then, the fact that power 4 corresponds to a transition in the beaviour of the problem reveals that the two-dimensional structure is emerging.

Qualitatively, the grid is two-dimensional on a large scale, and this fact must emerge when searching for low-mass ground states, since low-mass means widespread functions.

From a quantitative point of view, the emergence of the two-dimensional large scale structure occurs in the validity of the two-dimensional Sobolev inequality, i.e.

$$
\|u\|_{L^{2}(\mathcal{G})} \leq C\left\|u^{\prime}\right\|_{L^{1}(\mathcal{G})} \quad\left(u \in W^{1,1}(\mathcal{G})\right) .
$$

As well-known in Functional Analysis, such an inequality is typical of two-dimensional domains, whereas in one-dimension one has the one-dimensional Sobolev inequality

$$
\|u\|_{L^{\infty}(\mathcal{G})} \leq C\left\|u^{\prime}\right\|_{L^{1}(\mathcal{G})} \quad\left(u \in W^{1,1}(\mathcal{G})\right) .
$$

Now, inequality (9) is easy to prove for every one-dimensional non-compact graph, just using

$$
u(x)=\int_{\gamma} u^{\prime}(t) d t
$$

where $x$ is any point of the graph and the symbol $\gamma$ denotes a path isomorphic to a half-line starting at $x$. The existence of such a path is ensured by the fact that the graph is non-compact (therefore it extends up to infinity) and connected (so that it is possible to reach the infinity from $x$ through a sequence of adjacent edges).

It is then clear that what marks the transition between the one and the two-dimensional regime is the coexistence of (9) and (8), so that what really characterizes the grid, as well as every structure dysplaying a two-dimensional nature in the large scale, is the validity of (8).

As one shall expect, such a portrait can be generalized to the setting of periodic graphs exploiting higher dimensional structures in the large scale, like regular $n$-dimensional grids. In this context, it is readily seen that the dimensional crossover takes place between the one-dimensional and the $n$-dimensional critical power (see [30] for the explicit discussion of the case $n=3$ ).

In this paper we show that for the honeycomb graph, namely the grid made of the periodic repetition of a hexagon along a two-dimensional mesh (see Figure 2), estimate (8) holds true. Moving from this fact, we deduce a complete result about the existence or nonexistence of ground states, closely following the steps introduced in [29]. 


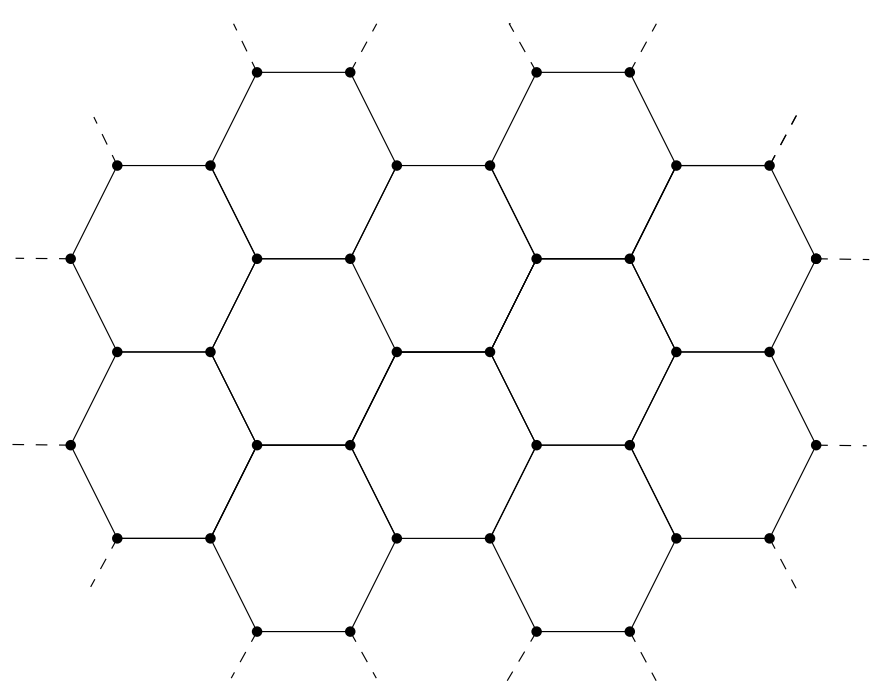

Figure 2. The infinite two-dimensional hexagonal grid $\mathcal{G}$.

\subsection{Existence of ground states in the honeycomb: the complete result}

Let us summarize the roadmap followed in [29], since for the sake of studying the hexagonal grid the steps will be the same. We shall therefore develop in detail only the part that differs significantly from the case of the square grid.

As explained in Section 1.1, our task is to prove the validity of a Sobolev inequality. This will be accomplished in Theorem 3.1. Once found the correct Sobolev inequality, and starting from it, we will prove another family of estimates, called Gagliardo-Nirenberg inequalities, that estimate the potential term in (3) by the product of suitable powers of the mass and of the kinetic energy.

In the case of functions on the line, as well as on general metric graphs, such estimates read as follows

$$
\|u\|_{L^{p}(\mathcal{G})}^{p} \leq C\left\|u^{\prime}\right\|_{L^{2}(\mathcal{G})}^{\frac{p}{2}-1}\|u\|_{L^{2}(\mathcal{G})}^{\frac{p}{2}+1}
$$

and, inserted in (3), give

$$
E(u, \mathcal{G}) \geq \frac{1}{2}\left\|u^{\prime}\right\|_{L^{2}(\mathcal{G})}^{2}-\frac{C}{p}\left\|u^{\prime}\right\|_{L^{2}(\mathcal{G})}^{\frac{p}{2}-1} \mu^{\frac{p}{4}+\frac{1}{2}}
$$

from which one immediately concludes that, if $2<p<6$, then

$$
\mathcal{E}(\mu)>-\infty
$$

that is a necessary condition for the existence of a ground state. In order to conclude for the existence, one should then prove the convergence of minimizing sequences. Let us just give some hint on how this proof may work. For details we refer the reader to [29]. First, to avoid that the minimizing sequence runs away, converging then to zero in the weak sense, one should localize the functions of the sequence. This is easily accomplished by exploiting the periodicity of the graph, by which, given a minimizing sequence, one defines a new minimizing sequence translating the elements of the old one in such a way that every function has its maximum on a fixed edge. Once excluded the possibility of escaping at infinity, the only way for a minimizing sequence in order not to converge is to spread along the grid, reaching in the limit zero energy.

As a consequence, in order to show that a minimizing sequence converges, it suffices to exhibit a function with negative energy.

The existence of a function with negative energy in the cases $2<p<4$ for every $\mu$, and $4 \leq p<6$ for $\mu$ large enough, is the content of Theorem 1.1 and of the positive part of point $(i)$ in Theorem 1.2. 
Conversely, in order to catch the core of the non-existence results at points (ii) and (iii) in Theorem 1.2 , let us consider inequality (10) and notice that for $p=6$ it specializes to

$$
\|u\|_{L^{6}(\mathcal{G})}^{6} \leq C\left\|u^{\prime}\right\|_{L^{2}(\mathcal{G})}^{2}\|u\|_{L^{2}(\mathcal{G})}^{4} .
$$

On the other hand, from (8) one derives

$$
\|u\|_{L^{p}(\mathcal{G})}^{p} \leq C\left\|u^{\prime}\right\|_{L^{2}(\mathcal{G})}^{p-2}\|u\|_{L^{2}(\mathcal{G})}^{2},
$$

that, for $p=4$, gives

$$
\|u\|_{L^{4}(\mathcal{G})}^{4} \leq C\left\|u^{\prime}\right\|_{L^{2}(\mathcal{G})}^{2}\|u\|_{L^{2}(\mathcal{G})}^{2} .
$$

Now, interpolating between (12) and (14) one has, for every $p \in[4,6]$

$$
\|u\|_{L^{p}(\mathcal{G})}^{p} \leq C\left\|u^{\prime}\right\|_{L^{2}(\mathcal{G})}^{2}\|u\|_{L^{2}(\mathcal{G})}^{p-2} .
$$

Then, by (15)

$$
\begin{aligned}
E(u, \mathcal{G}) & \geq \frac{1}{2}\left\|u^{\prime}\right\|_{L^{2}(\mathcal{G})}^{2}-\frac{C}{p}\left\|u^{\prime}\right\|_{L^{2}(\mathcal{G})}^{2}\|u\|_{L^{2}(\mathcal{G})}^{p-2} \\
& =\frac{1}{2}\left\|u^{\prime}\right\|_{L^{2}(\mathcal{G})}^{2}\left(1-\frac{2 C}{p} \mu^{\frac{p}{2}-1}\right)
\end{aligned}
$$

Then, for every $p \in[4,6]$ there exists a positive value $\mu_{p}>0$ given by

$$
\mu_{p}:=\left(\frac{p}{2 C}\right)^{\frac{2}{p-2}}
$$

with $C$ being the sharpest constant in (15), such that

- If $\mu<\mu_{p}$, then $E(u, \mathcal{G})>0$ for every $u \in H_{\mu}^{1}(\mathcal{G})$. Since, by spreading the function $u$ along the grid, one immediately gets $\mathcal{E}(\mu)=0$, it turns out that the infimum is not attained and ground states do not exist.

- If $\mu>\mu_{p}$ it turns out that $\mathcal{E}(\mu)<0$, and possibly $-\infty$.

The dimensional crossover lies exactly in this continuous transition from the subcritical regime (where for every mass there is a ground state) to the supercritical, where there are values of the mass in correspondence of which the energy is not lower bounded. In standard cases, such a transition only occurs in correspondence of the unique critical case, that amounts to 6 in dimension one, and to 4 in dimension two. In the case of a doubly periodic graph as the honeycomb we consider here, this actually takes place for all the nonlinearities $p$ between 4 and 6 , so that a continuum of critical exponents arises between the critical power of dimension 2 and the one of dimension 1.

Here are the complete results:

Theorem 1.1. Let $2<p<4$. Then, for every $\mu>0$, there exists a ground state of mass $\mu$.

Theorem 1.2. For every $p \in[4,6]$ there exists a critical mass $\mu_{p}>0$ such that

(i) if $p \in(4,6)$ then ground states of mass $\mu$ exist if and only if $\mu \geq \mu_{p}$, and

$$
\mathcal{E}(\mathcal{G}) \begin{cases}=0 & \text { if } \mu \leq \mu_{p} \\ <0 & \text { if } \mu>\mu_{p} .\end{cases}
$$

(ii) if $p=4$ then ground states of mass $\mu$ exist if $\mu>\mu_{4}$ and they do not exist if $\mu \leq \mu_{4}$. Furthermore, (17) holds true also in the case $p=4$. 
(iii) if $p=6$ then ground states never exist, independently of the value of $\mu$, and

$$
\mathcal{E}(\mu)= \begin{cases}0 & \text { if } \mu \leq \mu_{6} \\ -\infty & \text { if } \mu>\mu_{6}\end{cases}
$$

Theorems 1.1 and 1.2 do not differ from their analogues in the case of the square grid, treated in [29]. The only remarkable new procedures concern the proof of Sobolev inequality as in Theorem 3.1 and the construction of a function with negative energy proving the existence of a ground state in the regime $p \in(2,4)$.

The remainder of the paper is organised as follows. Section 2 sets some notation for the honeycomb, whereas Section 3 develops the proof of Sobolev inequality (9). Finally, in Section 4 we exhibit functions realizing strictly negative energy when $p \in(2,4)$, giving the proof of Theorem 1.1.

\section{Notation}

Before going further, a bit of notation is necessary. Particularly, to ease several of the upcoming arguments, it is useful to decompose the hexagonal grid in two family of parallel infinite paths, so that the whole graph $\mathcal{G}$ can be described as their union.

To this purpose, let us introduce the following construction. Fix any cell in $\mathcal{G}$ and denote by $o$ its lower left vertex. Note that, starting at $o$, there is one horizontal edge at the right and, at the left of $o$, an edge directed upwards and another one directed downwards. Consider then the infinite path running through $o$ constructed in this way. First, moving from $o$ to the right, follow the infinite path that alternates a horizontal and an upward edge. Then, moving from $o$ to the left, follow the infinite path that alternates a downward and a horizontal edge. We denote by $L_{0}$ the union of these two paths (see Figure 3(a)).

Similarly, consider both the infinite path that goes from $o$ to the left alternating an upward and a horizontal edge, and the one that originates at $o$ and moves to the right alternating a horizontal and a downward edge. We denote the union of these two by $R_{0}$ (see Figure $3(\mathrm{~b})$ ).

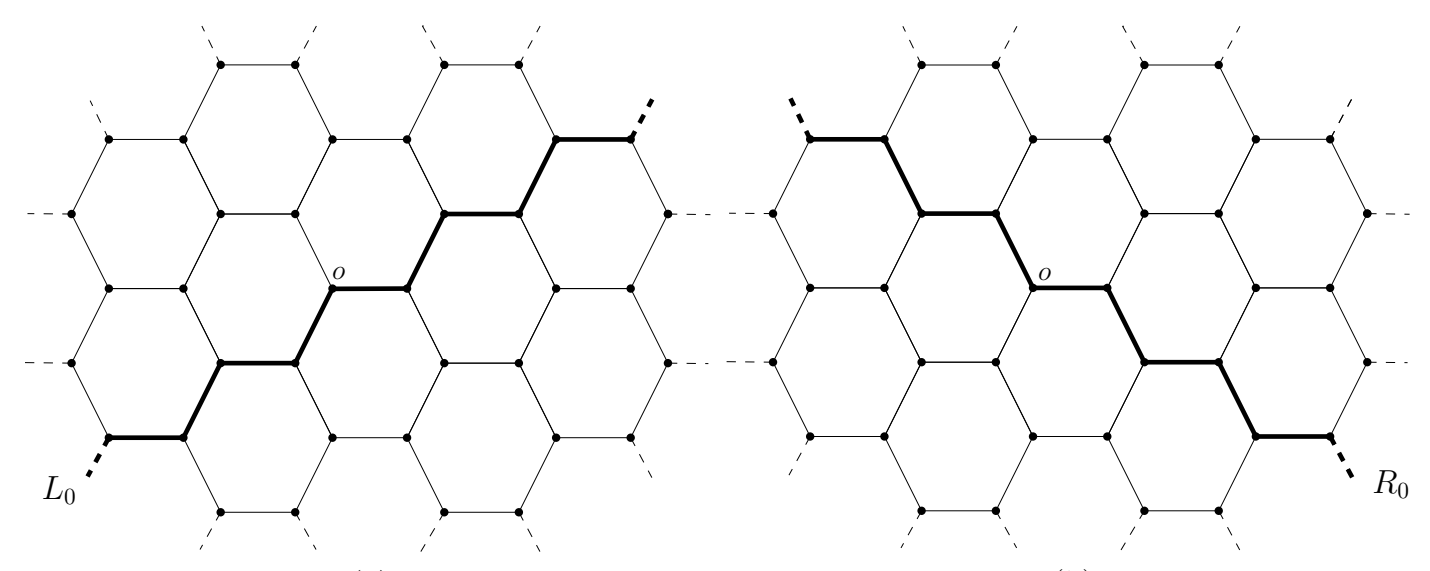

(a)

(b)

Figure 3. the paths $L_{0}(\mathrm{a})$ and $R_{0}(\mathrm{~b})$.

Note that both on $L_{0}$ and on $R_{0}$ natural coordinates $x_{L_{0}}: L_{0} \rightarrow(-\infty,+\infty), x_{R_{0}}: R_{0} \rightarrow(-\infty,+\infty)$ can be defined, so that they can be identified with real lines with the origin in $o$.

Now, consider for instance the vertex belonging to $L_{0}$ which is at distance $2 \ell$ (measured along $\mathcal{G}$ ) from $o$ on its right. It is immediate to see that an infinite path running through this vertex and parallel to $R_{0}$ can be recovered by repeating the procedure used to construct $R_{0}$. However, this is not the case if we consider the vertex of $L_{0}$ at distance $\ell$ from $o$ on its right, as it already belongs to $R_{0}$.

More generally, through every vertex on $L_{0}$ located at an even distance from $o$ on its right runs an infinite path parallel to $R_{0}$. It is then straightforward to check that the same holds true also for every vertex on $L_{0}$ located at an odd (in terms of $\ell$ ) distance from $o$ at its left (whereas vertices at even distances 
on the left do not provide any additional path). This leads to a family $\left\{R_{j}\right\}_{j \in \mathbb{Z}}$ of infinite parallel paths in $\mathcal{G}$.

Analogously, one can consider the family of infinite paths $\left\{L_{i}\right\}_{i \in \mathbb{Z}}$ all parallel to $L_{0}$, constructed by taking any vertex on $R_{0}$ either at an even distance from $o$ at its right or at odd distance from $o$ at its left and repeating the steps in the construction of $L_{0}$.

We stress the fact that the set defined by $\left(\bigcup_{i \in \mathbb{Z}} L_{i}\right) \cap\left(\bigcup_{j \in \mathbb{Z}} R_{j}\right)$ is composed by all the horizontal edges of $\mathcal{G}$ and for this reason it follows

$$
\mathcal{G} \subset\left(\bigcup_{i \in \mathbb{Z}} L_{i}\right) \cup\left(\bigcup_{j \in \mathbb{Z}} R_{j}\right)
$$

In particular $L_{i} \cap R_{j} \neq \emptyset$ for every $i, j \in \mathbb{Z}$, as they share exactly one horizontal edge.

Finally, given $i, j \in \mathbb{Z}$, we denote by $I_{i}^{j} \subset L_{i}$ the union of the horizontal edge that $L_{i}$ shares with $R_{j}$ and the upward edge on its right. Moreover, we call $v_{i}^{j}$ the first vertex of $I_{i}^{j}$ that we meet walking down $R_{j}$ from $-\infty$ (see Figure 4(a)). Note that, for every $i, L_{i}=\bigcup_{j \in \mathbb{Z}} I_{i}^{j}$. Similarly, we define $J_{j}^{i}$ as the union of the horizontal edge shared by $L_{i}$ and $R_{j}$ and the upward edge at its left. As before, we observe that, for every $j \in \mathbb{Z}, R_{j}=\bigcup_{i \in \mathbb{Z}} J_{j}^{i}$ and again we denote by $w_{j}^{i}$ the first vertex of $J_{j}^{i}$ that we encounter walking through $L_{i}$ from $-\infty$ (Figure $4(\mathrm{~b})$ ).

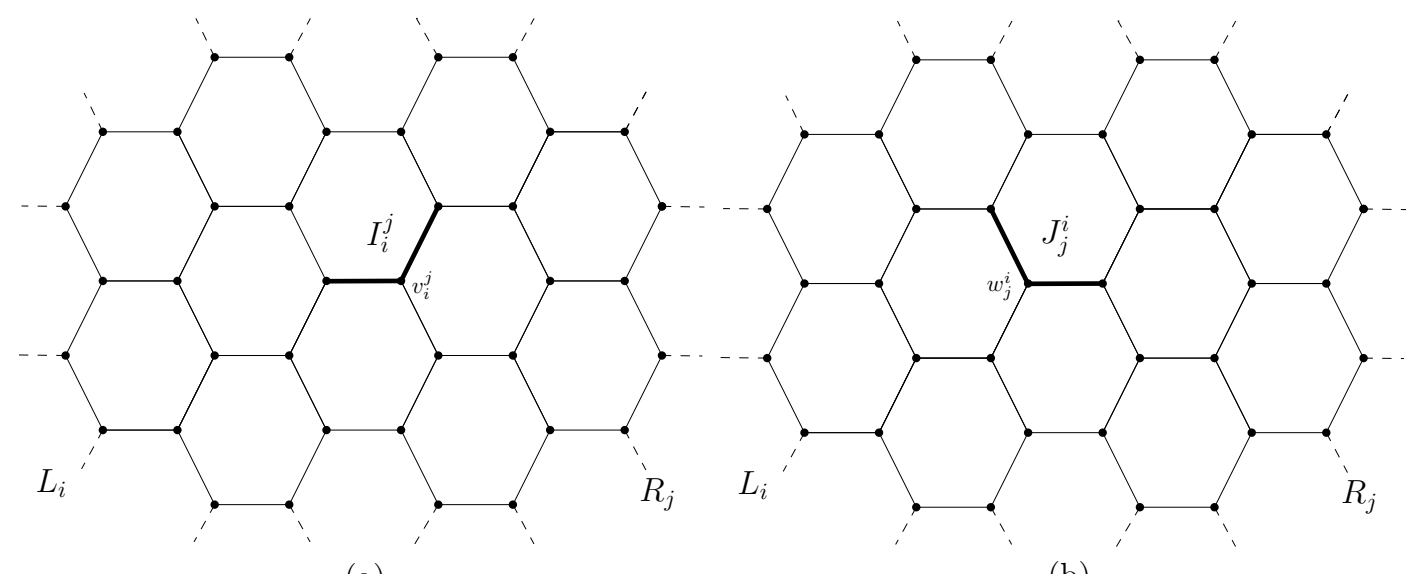

(a)

(b)

Figure 4. The subsets $I_{i}^{j}(\mathrm{a})$ and $J_{j}^{i}(\mathrm{~b})$.

\section{Sobolev inequality}

This section is devoted to the derivation of some functional inequalities that describe in which sense the hexagonal grid graph $\mathcal{G}$ interpolates between one-dimensional and two-dimensional behaviour. Particularly, the two-dimensional nature of the graph shows up explicitly with the following result, stating the validity of the Sobolev inequality in the form typical of dimension two.

Theorem 3.1. For every $u \in W^{1,1}(\mathcal{G})$,

$$
\|u\|_{L^{2}(\mathcal{G})} \leq 2 \sqrt{2 l}\left\|u^{\prime}\right\|_{L^{1}(\mathcal{G})} .
$$

Proof. We beforehand remind that $\mathcal{G} \subset\left(\bigcup_{i \in \mathbb{Z}} L_{i}\right) \cup\left(\bigcup_{j \in \mathbb{Z}} R_{j}\right)$, so that

$$
\|u\|_{L^{2}(\mathcal{G})}^{2} \leq \sum_{i}\|u\|_{L^{2}\left(L_{i}\right)}^{2}+\sum_{j}\|u\|_{L^{2}\left(R_{j}\right)}^{2} .
$$

In order to prove (19), we aims at estimating the two terms on the right side of (20). Let us start with $\sum_{i}\|u\|_{L^{2}\left(L_{i}\right)}^{2}$, where $\|u\|_{L^{2}\left(L_{i}\right)}^{2}=\int_{L_{i}}|u(x)|^{2} d x$. 
Consider any point $x \in \mathcal{G}$ located on $L_{i}$. Observe that $x$ can be reached following at least two different paths on $\mathcal{G}$. The first one walks through $L_{i}$ from $-\infty$ to $x$, whereas the second one runs through $R_{j}$ from $-\infty$ to the vertex $v_{i}^{j}$ and then moves on $L_{i}$ from $v_{i}^{j}$ to $x$ (Figure 5). Identifying with some abuse of notation the points $x$ and $v_{i}^{j}$ with their corresponding coordinates $x_{L_{i}}(x), x_{L_{i}}\left(v_{i}^{j}\right)$ and $x_{R_{j}}\left(v_{i}^{j}\right)$, we denote by $L_{i}(-\infty, x), R_{j}\left(-\infty, v_{i}^{j}\right)$ and $L_{i}\left(v_{i}^{j}, x\right)$ the paths from $-\infty$ to $x$ along $L_{i}$, from $-\infty$ to $v_{i}^{j}$ along $R_{j}$ and from $v_{i}^{j}$ to $x$ along $L_{i}$, respectively.

Thus, we get

$$
u(x)=\int_{L_{i}(-\infty, x)} u^{\prime}(\tau) d \tau
$$

and

$$
u(x)=\int_{R_{j}\left(-\infty, v_{i}^{j}\right)} u^{\prime}(\tau) d \tau+\int_{L_{i}\left(v_{i}^{j}, x\right)} u^{\prime}(\tau) d \tau .
$$

Multiplying (21) and (22) and using the fact that $L_{i}(-\infty, x) \subset L_{i}, R_{j}\left(-\infty, v_{i}^{j}\right) \subset R_{j}$ and $L_{i}\left(v_{i}^{j}, x\right) \subset I_{i}^{j}$, we estimate

$$
\begin{aligned}
|u(x)|^{2} & =\left|\int_{L_{i}(-\infty, x)} u^{\prime}(\tau) d \tau\right| \cdot\left|\int_{R_{j}\left(-\infty, v_{i}^{j}\right)} u^{\prime}(\tau) d \tau+\int_{L_{i}\left(v_{i}^{j}, x\right)} u^{\prime}(\tau) d \tau\right| \\
& \leq\left(\int_{L_{i}(-\infty, x)}\left|u^{\prime}(\tau)\right| d \tau\right) \cdot\left(\int_{R_{j}\left(-\infty, v_{i}^{j}\right)}\left|u^{\prime}(\tau)\right| d \tau+\int_{L_{i}\left(v_{i}^{j}, x\right)}\left|u^{\prime}(\tau)\right| d \tau\right) \\
& \leq\left(\int_{L_{i}}\left|u^{\prime}(\tau)\right| d \tau\right) \cdot\left(\int_{R_{j}}\left|u^{\prime}(\tau)\right| d \tau+\int_{I_{i}^{j}}\left|u^{\prime}(\tau)\right| d \tau\right) .
\end{aligned}
$$

Then, integrating on $L_{i}$

$$
\int_{L_{i}}|u(x)|^{2} d x=\int_{L_{i}}\left|u^{\prime}(\tau)\right| d \tau\left(\int_{L_{i}}\left(\int_{R_{j}}\left|u^{\prime}(\tau)\right| d \tau+\int_{I_{i}^{j}}\left|u^{\prime}(\tau)\right| d \tau\right) d x\right) .
$$

Recall that $L_{i}=\bigcup_{j \in \mathbb{Z}} I_{i}^{j}$ and note that both $\int_{R_{j}}\left|u^{\prime}(\tau)\right| d \tau$ and $\int_{I_{i}^{j}}\left|u^{\prime}(\tau)\right| d \tau$ are piecewise constant on each $I_{i}^{j}$ as functions of $x$. Hence, there results

$$
\int_{L_{i}}\left(\int_{R_{j}}\left|u^{\prime}(\tau)\right| d \tau\right) d x=2 l \sum_{j \in \mathbb{Z}} \int_{R_{j}}\left|u^{\prime}(\tau)\right| d \tau
$$

and

$$
\int_{L_{i}}\left(\int_{I_{i}^{j}}\left|u^{\prime}(\tau)\right| d \tau\right) d x=2 l \sum_{j \in \mathbb{Z}} \int_{I_{i}^{j}}\left|u^{\prime}(\tau)\right| d \tau=2 l \int_{L_{i}}\left|u^{\prime}(\tau)\right| d \tau .
$$

By (23), (24) and (25) it follows

$$
\begin{aligned}
\int_{L_{i}}|u(x)|^{2} d x & =\int_{L_{i}}\left|u^{\prime}(\tau)\right| d \tau\left(2 l \sum_{j \in \mathbb{Z}} \int_{R_{j}}\left|u^{\prime}(\tau)\right| d \tau+2 l \int_{L_{i}}\left|u^{\prime}(\tau)\right| d \tau\right) \\
& \leq 4 l\left\|u^{\prime}\right\|_{L^{1}(\mathcal{G})} \int_{L_{i}}\left|u^{\prime}(\tau)\right| d \tau
\end{aligned}
$$

as each term in the sum can be dominated by $\left\|u^{\prime}\right\|_{L^{1}(\mathcal{G})}$.

Finally, summing over $i \in \mathbb{Z}$ yields

$$
\sum_{i \in \mathbb{Z}} \int_{L_{i}}|u(x)|^{2} \leq 4 l\left\|u^{\prime}\right\|_{L^{1}(\mathcal{G})} \sum_{i \in \mathbb{Z}} \int_{L_{i}}\left|u^{\prime}(\tau)\right| d \tau \leq 4 l\left\|u^{\prime}\right\|_{L^{1}(\mathcal{G})}^{2} .
$$




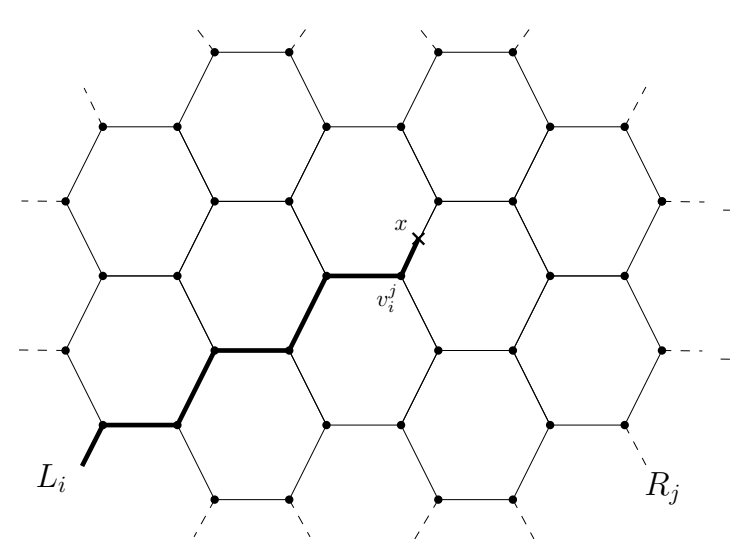

(a)

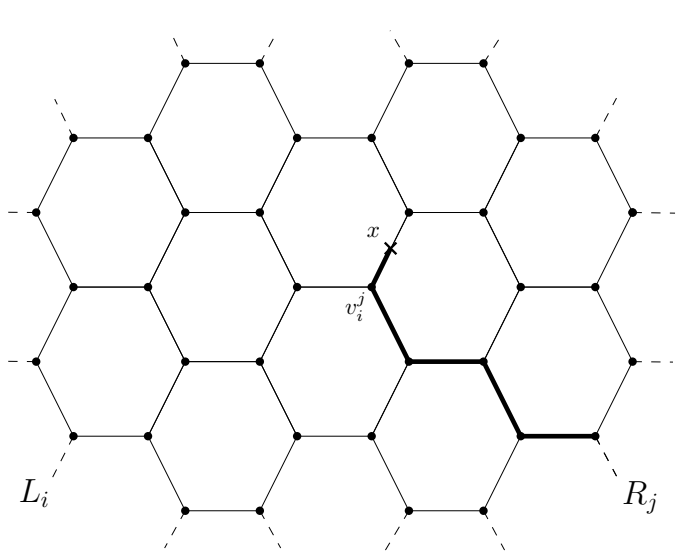

(b)

Figure 5. The paths from $-\infty$ to $x$ along $L_{i}(\mathrm{a})$ and $R_{j}(\mathrm{~b})$ as in the proof of Theorem 3.1.

The same procedure can be adapted to estimate $\sum_{j \in \mathbb{Z}} \int_{R_{j}}|u(x)|^{2} d x$, replacing $I_{i}^{j}$ with $J_{j}^{i}$ whenever needed, so that by (20) we end up with

$$
\|u\|_{L^{2}(\mathcal{G})}^{2} \leq 8 \ell\left\|u^{\prime}\right\|_{L^{1}(\mathcal{G})}^{2} .
$$

Arguing as in the proof of Theorem 2.3 in [29], it can then be proved that Theorem 3.1 entails the following two-dimensional Gagliardo-Nirenberg inequality on $\mathcal{G}$

$$
\|u\|_{L^{p}(\mathcal{G})}^{p} \leq C\|u\|_{L^{2}(\mathcal{G})}^{2}\left\|u^{\prime}\right\|_{L^{2}(\mathcal{G})}^{p-2}
$$

for every $u \in H^{1}(\mathcal{G})$ and $p \geq 2$ (here $C$ denotes a universal constant).

On the other hand, as for every non-compact metric graph, it is known that also the one-dimensional Gagliardo-Nirenberg inequality

$$
\|u\|_{L^{p}(\mathcal{G})}^{p} \leq\|u\|_{L^{2}(\mathcal{G})}^{\frac{p}{2}+1}\left\|u^{\prime}\right\|_{L^{2}(\mathcal{G})}^{\frac{p}{2}-1}
$$

holds true on $\mathcal{G}$, again for every $u \in H^{1}(\mathcal{G})$ and $p \geq 2$ (for a simple proof relying on the theory of rearrangements on graphs see for instance [31]).

Hence, combining (26)-(27), a new version of the Gagliardo-Nirenberg inequality can be derived, which we refer to as interpolated Gagliardo-Nirenberg inequality, that accounts for the dimensional crossover in Theorem 1.2. Indeed, for every $p \in[4,6]$ there exists a constant $K_{p}$, depending only on $p$, such that

$$
\|u\|_{L^{p}(\mathcal{G})}^{p} \leq K_{p}\|u\|_{L^{2}(\mathcal{G})}^{p-2}\left\|u^{\prime}\right\|_{L^{2}(\mathcal{G})}^{2}
$$

for every $u \in H^{1}(\mathcal{G})$ (as the argument is the same, we refer to Corollary 2.4 in [29] for a complete proof of this fact).

\section{Existence result: proof of Theorem 1.1}

In this section, we provide the proof of Theorem 1.1, showing that if $p$ is smaller than 4 , then ground states always exist for every value of the mass.

To this purpose, we first recall a general compactness result, originally proved in Proposition 3.3 of [29], which is valid for every doubly periodic metric graphs, so that it also applies in the case of the two-dimensional hexagonal grid we are dealing with.

Proposition 4.1 (Proposition 3.3, [29]). Let $p<6$ and $\mu<0$. If $\mathcal{E}(\mu)<0$, then a ground state with mass $\mu$ exists. 
Proof of Theorem 1.1. In view of Proposition 4.1, given $\mu>0$, it is enough to prove that $\mathcal{E}(\mu)<0$ to show that ground states in $H_{\mu}^{1}(\mathcal{G})$ exist.

We consider the following construction. For every $i \in \mathbb{Z}$, recall that $L_{i}$ is identified with a real line $(-\infty,+\infty)$ through a coordinate $x_{L_{i}}$, and we are free to choose which vertex $\mathrm{V} \in L_{i}$ corresponds to the origin $x_{L_{i}}(\mathrm{v})=0$. We thus fix the origin of each $L_{i}$ in the following way. First, set the origin of $L_{0}$ at any of its vertices being the left endpoint of a horizontal edge. Then, since the upward edge on the left of this vertex connects $L_{0}$ with $L_{1}$, set the origin of $L_{1}$ at the endpoint of this bridging edge. Let then $\bar{L}_{0}$ be the straight line in the plane passing through both the origin of $L_{0}$ and the one of $L_{1}$. For each $i \in \mathbb{Z}, \bar{L}_{0}$ intersects $L_{i}$ in exactly one vertex of $\mathcal{G}$, so that we set this point to be the origin of $L_{i}$.

Note that the intersection of $\bar{L}_{0}$ with the whole grid $\mathcal{G}$ is a disjoint union of edges, each joining a couple of paths $L_{i}, L_{i+1}$, for some $i \in \mathbb{Z}$. Precisely, we write

$$
\bar{L}_{0} \cap \mathcal{G}=\bigsqcup_{i \in \mathbb{Z}} b_{2 i}^{0}
$$

where, given $i \in \mathbb{Z}, b_{2 i}^{0}$ denotes the bridging edge between $L_{2 i}$ and $L_{2 i+1}$ that belongs to $\bar{L}_{0}$.

Similarly, for every $k \in \mathbb{Z}$, let $\bar{L}_{k}$ be the straight line in the plane parallel to $\bar{L}_{0}$ passing through the vertex of $\mathrm{v} \in L_{0}$ corresponding to $x_{L_{0}}(\mathrm{v})=k$, so that

$$
\bar{L}_{k} \cap \mathcal{G}= \begin{cases}\bigsqcup_{i \in \mathbb{Z}} b_{2 i}^{k} & \text { if } k \text { even } \\ \bigsqcup_{i \in \mathbb{Z}} b_{2 i-1}^{k} & \text { if } k \text { odd }\end{cases}
$$

where again $b_{2 i}^{k}$ (resp. $b_{2 i-1}^{k}$ ) is the edge of $\mathcal{G}$ joining $L_{2 i}$ with $L_{2 i+1}$ (resp. $L_{2 i-1}$ with $L_{2 i}$ ) that belongs to $\bar{L}_{k}$.

Moreover, identifying each $b_{j}^{k}$ with the interval $[0,1]$ through the coordinate $x_{b_{j}^{k}}: b_{j}^{k} \rightarrow[0,1]$, we use the following notation: if $j \geq 0$, then we set $x_{b_{j}^{k}}(\mathrm{v})=0$ for $\mathrm{v}=b_{j}^{k} \cap L_{j}$, whereas if $j<0$, then we set $x_{b_{j}^{k}}(0)=\mathrm{v}$ for $\mathrm{v}=b_{j}^{k} \cap L_{j+1}$.

Then, given $\varepsilon>0$, we define (see Figure 6 )

$$
u_{\varepsilon}(x):= \begin{cases}e^{-\varepsilon(|x|+|i|)} & \text { if } x \in L_{i}, \text { for some } i \in \mathbb{Z} \\ e^{-\varepsilon(|x|+|i|+j)} & \text { if } x \in b_{j}^{i}, \text { for some } j, k \in \mathbb{Z}, j \geq 0 \\ e^{-\varepsilon(|x|+|i|+|j+1|)} & \text { if } x \in b_{j}^{i}, \text { for some } j, k \in \mathbb{Z}, j<0 .\end{cases}
$$

By construction, $u \in H^{1}(\mathcal{G})$ and, given $i \in \mathbb{Z}$,

$$
\begin{aligned}
\int_{L_{i}}\left|u_{\varepsilon}\right|^{p} d x & =2 \int_{0}^{+\infty} e^{-p \varepsilon(x+|i|)} d x=\frac{2 e^{-p \varepsilon|i|}}{p \varepsilon} \\
\int_{\bar{L}_{i} \cap \mathcal{G}}\left|u_{\varepsilon}\right|^{p} d x & =\int_{0}^{+\infty} e^{-p \varepsilon(x+|i|)} d x=\frac{e^{-p \varepsilon|i|}}{p \varepsilon}
\end{aligned}
$$

for every $p \geq 2$ and

$$
\begin{gathered}
\int_{L_{i}}\left|u_{\varepsilon}^{\prime}\right|^{2} d x=2 \varepsilon^{2} \int_{0}^{+\infty} e^{-2 \varepsilon(|x|+|i|)} d x=\varepsilon e^{-2 \varepsilon|i|} \\
\int_{\bar{L}_{i} \cap \mathcal{G}}\left|u_{\varepsilon}^{\prime}\right|^{2} d x=\varepsilon^{2} \int_{0}^{+\infty} e^{-2 \varepsilon(x+|i|)} d x=\frac{\varepsilon e^{-2 \varepsilon|i|}}{2} .
\end{gathered}
$$

Since $\mathcal{G}=\left(\bigcup_{i \in \mathbb{Z}} L_{i}\right) \cup\left(\bigcup_{i \in \mathbb{Z}} \bar{L}_{i} \cap \mathcal{G}\right)$, we get 


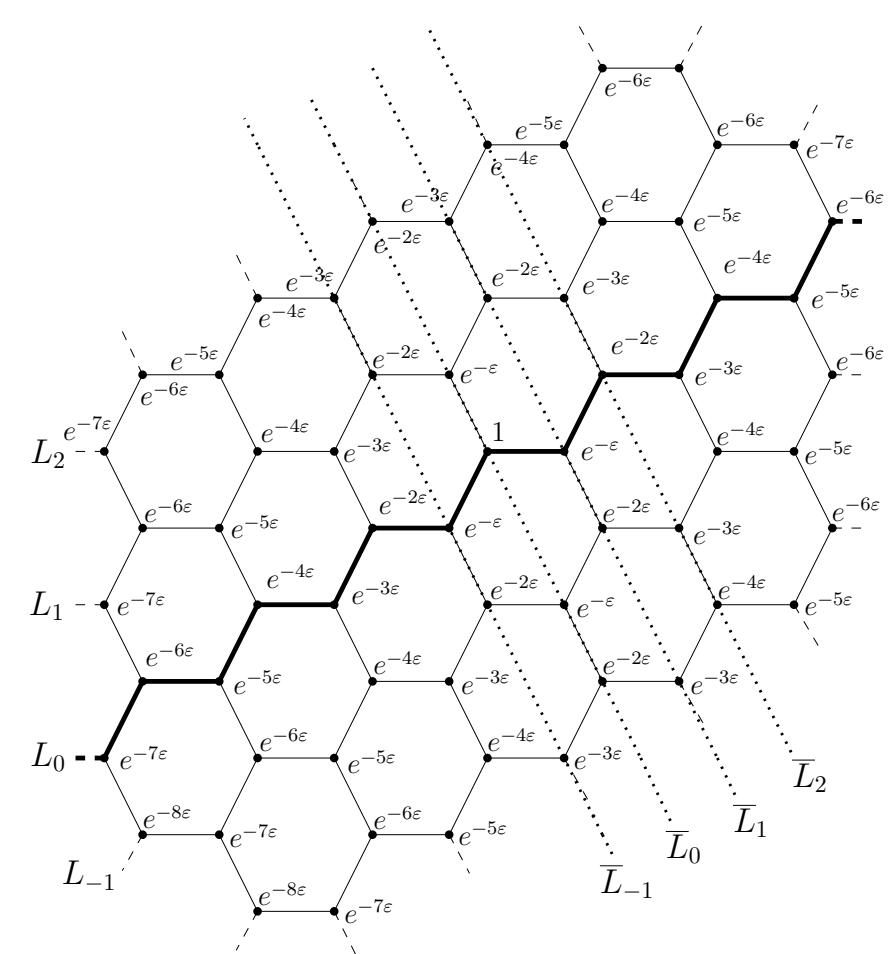

Figure 6. the construction of the function $u$ in the proof of Theorem 1.1, with the straight lines $\bar{L}_{i}$ and the values of $u$ at the vertices of $\mathcal{G}$.

$$
\begin{aligned}
& \int_{\mathcal{G}}\left|u_{\varepsilon}\right|^{p} d x=\sum_{i \in \mathbb{Z}} \int_{L_{i}}\left|u_{\varepsilon}\right|^{p} d x+\sum_{i \in \mathbb{Z}} \int_{\bar{L}_{i} \cap \mathcal{G}}\left|u_{\varepsilon}\right|^{p} d x=3\left(\frac{1}{p \varepsilon}+2 \sum_{i=1}^{\infty} \frac{e^{-p \varepsilon i}}{p \varepsilon}\right)=\frac{3\left(e^{p \varepsilon}+1\right)}{p \varepsilon\left(e^{p \varepsilon}-1\right)} \\
& \int_{\mathcal{G}}\left|u_{\varepsilon}^{\prime}\right|^{2} d x=\sum_{i \in \mathbb{Z}} \int_{L_{i}}\left|u_{\varepsilon}^{\prime}\right|^{2} d x+\sum_{i \in \mathbb{Z}} \int_{\bar{L}_{i} \cap \mathcal{G}}\left|u_{\varepsilon}^{\prime}\right|^{2} d x=3\left(\frac{\varepsilon}{2}+\sum_{i=1}^{\infty} \varepsilon e^{-2 \varepsilon i}\right)=\frac{3 \varepsilon\left(e^{2 \varepsilon}+1\right)}{2\left(e^{2 \varepsilon}-1\right)} .
\end{aligned}
$$

Hence, setting

$$
k_{\varepsilon}:=\left(\frac{2 \varepsilon\left(e^{2 \varepsilon}-1\right)}{3\left(e^{2 \varepsilon}+1\right)} \mu\right)^{1 / 2}
$$

and letting

$$
v_{\varepsilon}(x):=k_{\varepsilon} u_{\varepsilon}(x) \quad \forall x \in \mathcal{G}
$$

yields

$$
\left\|v_{\varepsilon}\right\|_{L^{2}(\mathcal{G})}^{2}=k_{\varepsilon}^{2} \int_{\mathcal{G}}\left|u_{\varepsilon}\right|^{2} d x=\mu .
$$

Therefore, $v_{\varepsilon} \in H_{\mu}^{1}(\mathcal{G})$ for every $\varepsilon>0$ and, taking advantage of the explicit formula above, as $\varepsilon \rightarrow 0$

$$
E\left(v_{\varepsilon}, \mathcal{G}\right)=\frac{1}{2} k_{\varepsilon}^{2} \int_{\mathcal{G}}\left|u_{\varepsilon}^{\prime}\right|^{2} d x-\frac{1}{p} k_{\varepsilon}^{p} \int_{\mathcal{G}}\left|u_{\varepsilon}\right|^{p} d x \sim \frac{1}{2} \mu \varepsilon^{2}-\frac{1}{p} C \mu^{p / 2} \varepsilon^{p-2}
$$

for some $C>0$ depending only on $p$. Thus, whenever $p<4$ and $\varepsilon$ is small enough, we have

$$
\mathcal{E}(\mu) \leq E\left(v_{\varepsilon}, \mathcal{G}\right)<0
$$

and we conclude. 


\section{Quantum graphs and dimensional crossover: the honeycomb}

\section{References}

1. K. Ruedenberg and C. W. Scherr, Free-electron network model for conjugated systems. I. Theory, The Journal of Chemical Physics, vol. 21, no. 9, pp. 1565-1581, 1953.

2. F. A. Mehmeti, Nonlinear Waves in Networks. Mathematical Research Series, Akademie Verlag, 1994.

3. R. Adami, C. Cacciapuoti, D. Finco, and D. Noja, Fast solitons on star graphs, Reviews in Mathematical Physics, vol. 23, no. 04, pp. 409-451, 2011.

4. M. Keel and T. Tao, Endpoint Strichartz estimates, American Journal of Mathematics, vol. 120, no. 5, pp. 955-980, 1998.

5. C. Kenig and F. Merle, Global well-posedness, scattering and blow-up for the energy critical, focusing non-linear Schrödinger equation in the radial case, Inventiones mathematicae, vol. 166, no. 3, pp. 645$675,2006$.

6. S. N. Bose, Plancks gesetz und lichtquantenhypothese, Zeitschrift für Physik, vol. 26, pp. 178-181, Dec 1924.

7. A. Einstein, Quantentheorie des einatomigen idealen Gases. No. 2 in Sitzungsberichte der Preussischen Akademie der Wissenschaften. Physikalisch-mathematische Klasse, Verlag d. Akad. d. Wiss., 1924.

8. T. Cazenave, Semilinear Schrödinger Equations. Courant lecture notes in mathematics, American Mathematical Society, 2003.

9. T. Cazenave and P. L. Lions, Orbital stability of standing waves for some nonlinear Schrödinger equations, Communications in Mathematical Physics, vol. 85, no. 4, pp. 549-561, 1982.

10. V. E. Zakharov and B. Shabat, Exact theory of two-dimensional self-focusing and one-dimensional self-modulation of waves in nonlinear media, Journal of Experimental and Theoretical Physics, vol. 34, no. 1 , pp. 62-71, 1972.

11. R. Adami, C. Cacciapuoti, D. Finco, and D. Noja, On the structure of critical energy levels for the cubic focusing NLS on star graphs, Journal of Physics A: Mathematical and Theoretical, vol. 45, p. 192001, apr 2012.

12. R. Adami, E. Serra, and P. Tilli, NLS ground states on graphs, Calculus of Variations and Partial Differential Equations, vol. 54, pp. 743-761, Sep 2015.

13. S. Dovetta and L. Tentarelli, Ground states of the $L^{2}$-critical NLS equation with localized nonlinearity on a tadpole graph, Operator Theory: Advances and Applications. to appear.

14. J. L. Marzuola and D. E. Pelinovsky, Ground state on the dumbbell graph, Applied Mathematics Research eXpress, vol. 2016, no. 1, pp. 98-145, 2016.

15. D. Noja, D. Pelinovsky, and G. Shaikhova, Bifurcations and stability of standing waves in the nonlinear Schrödinger equation on the tadpole graph, Nonlinearity, vol. 28, no. 7, pp. 2343-2378, 2015.

16. C. Cacciapuoti, S. Dovetta, and E. Serra, Variational and stability properties of constant solutions to the NLS equation on compact metric graphs, Milan Journal of Mathematics, vol. 86, no. 2, pp. 305$327,2018$.

17. S. Dovetta, Existence of infinitely many stationary solutions of the $L^{2}$-subcritical and critical NLSE on compact metric graphs, Journal of Differential Equations, vol. 264, no. 7, pp. 4806-4821, 2018.

18. S. Dovetta and L. Tentarelli, $L^{2}$-critical NLS on noncompact metric graphs with localized nonlinearity: topological and metric features, Calculus of Variations and Partial Differential Equations, 2019. to appear.

19. E. Serra and L. Tentarelli, Bound states of the NLS equation on metric graphs with localized nonlinearities, Journal of Differential Equations, vol. 260, no. 7, pp. 5627-5644, 2016.

20. E. Serra and L. Tentarelli, On the lack of bound states for certain NLS equations on metric graphs, Nonlinear Analysis: Theory, Methods and Applications, vol. 145, pp. 68-82, 2016.

21. L. Tentarelli, NLS ground states on metric graphs with localized nonlinearities, Journal of Mathematical Analysis and Applications, vol. 433, no. 1, pp. 291-304, 2016.

22. R. Adami, E. Serra, and P. Tilli, Negative energy ground states for the $L^{2}$-critical NLSE on metric 
graphs, Communications in Mathematical Physics, vol. 352, pp. 387-406, May 2017.

23. W. Borrelli, R. Carlone, and L. Tentarelli, Nonlinear Dirac equation on graphs with localized nonlinearities: Bound states and nonrelativistic limit, SIAM Journal on Mathematical Analysis, vol. 51, pp. 1046-1081, 012019.

24. W. Borrelli, R. Carlone, and L. Tentarelli, An overview on the standing waves of nonlinear Schrödinger and Dirac equations on metric graphs with localized nonlinearity, Symmetry, vol. 11, no. 2, 2019.

25. S. Gilg, D. Pelinovsky, and G. Schneider, Validity of the NLS approximation for periodic quantum graphs, Nonlinear Differential Equations and Applications NoDEA, vol. 63, no. 6, p. 30, 2016.

26. A. Pankov, Nonlinear Schrödinger equations on periodic metric graphs, Discrete e Continuous Dynamical Systems, vol. 38, no. 2, pp. 697-714, 2018.

27. D. Pelinovsky and G. Schneider, Bifurcations of standing localized waves on periodic graphs, Annales Henri Poincaré, vol. 18, no. 4, pp. 1185-1211, 2017.

28. S. Dovetta, Mass-constrained ground states of the stationary NLSE on periodic metric graphs, Nonlinear Differential Equations and Applications, 2019. to appear.

29. R. Adami, S. Dovetta, E. Serra, and P. Tilli, Dimensional crossover with a continuum of critical exponents for NLS on doubly periodic metric graphs, Analysis \& PDE, vol. 12, pp. 1597-1612, 02 2019.

30. R. Adami and S. Dovetta, One-dimensional versions of three-dimensional system: Ground states for the NLS on the spatial grid, Rendiconti di Matematica e delle sue Applicazioni, vol. 39, pp. 181-194, 2018.

31. R. Adami, E. Serra, and P. Tilli, Threshold phenomena and existence results for NLS ground states on metric graphs, Journal of Functional Analysis, vol. 271, no. 1, pp. 201 - 223, 2016. 